Review Article

\section{Veterinary vaccine development: The helical project}

\author{
lyasu Angani Dereja* \\ Animal Products, Veterinary Drug and Feed Quality Assessment Centre, Ethiopia
}

\section{Summary}

Vaccine production process have been fuzzy journey to the public and, in some degrees, to those in the setting. By clearly showing the lengthy and challenging journey of vaccine development process, thereby suggesting the economic and health implication of improper use of veterinary vaccines, the paper tries to add the attention given to infection prevention. Starting from the foundations, the types and requirements of veterinary vaccines are described. The paper concludes with current research and regulatory quos in the topic.

\section{Principles of vaccine immunity}

\section{What is vaccine}

Due to the global attention to infectious diseases, focus for vaccine development is rising parallelly. From infections that existed for decades to the current outbreaks, from local and national health officials to international organs like the World Health Organization (WHO), from biomedical students to the world class researchers, vaccine and vaccine development is being a topic of relief everywhere. Due to variations of regulatory lexicons and some differences in medical jargon among different areas, there still exist some sort of variations of understanding the preliminaries of vaccine including its medical taxonomy. Some people say it as vaccine, others as immunization and still others, assume it as some sort of therapy. Before going further deeper, it is worth to clear those fuzzy areas.

Is vaccine a drug? Many people consider vaccine as a treatment for diseases. Although vaccines are significant focus of the medicine as a field, technically speaking, vaccine is way different from treatment - it is a tool that motivates and enable body of animals (and humans, of course) from diseases of infectious nature [1]. A vaccine "stimulates your immune system to produce antibodies, exactly like it would if you were exposed to the disease". This may be translated to training the body's immune system to be able to fight external invaders. This is through administration of the same germ that causes the specific disease we are administering a vaccine to. However, they have to be either killed or weakened to the point that they don't make the host sick.

It is due to this fact that vaccines are usually categorized

\section{More Information}

\author{
*Address for Correspondence: lyasu Angani \\ Dereja, Animal Products, Veterinary Drug and \\ Feed Quality Assessment Centre, Addis Ababa, \\ Ethiopia, Tel: +251910869263; \\ Email: iyasuangani@gmail.com \\ Submitted: 18 August 2020 \\ Approved: 27 August 2020 \\ Published: 28 August 2020 \\ How to cite this article: Dereja IA. Veterinary \\ vaccine development: The helical project. \\ Insights Vet Sci. 2020; 4: 042-047.
}

DOI: 10.29328/journal.ivs.1001025

ORCiD: orcid.org/0000-0001-8829-93071

Copyright: ๑ 2020 Dereja IA. This is an open access article distributed under the Creative Commons Attribution License, which permits unrestricted use, distribution, and reproduction in any medium, provided the original work is properly cited.

Keywords: Veterinary; Vaccine; Development; Ethiopia; Animal

\section{(D) Check for updates}

OPEN ACCESS as biologicals. Biologicals are those medical tools driven from or related to living organisms [2]. In the words of the WHO, Biologicals are "are those class of medicines which are grown and then purified from large-scale cell cultures of bacteria or yeast, or plant or animal cells" [3]. This suggests us that welldeveloped knowledge of the causative agent of the disease we are attempting to prevent using vaccine is the fundamental prerequisite to think about a certain vaccine.

A definition from Winchester Hospital [4] summarizes our explanation:

A vaccine is a biological substance designed to protect humans from infections caused by bacteria and viruses. Vaccines are also called immunizations because they take advantage of our natural immune system's ability to prevent infectious illness. To understand how vaccines work, we need to consider how our immune system protects us from infections.

\section{Types of vaccine}

As true to other medical supplies, vaccines generally are classified into several categories based on certain criterial 
related to the technical process of development or approach, formulation and the specific source of the substance. Veterinary vaccines are not exceptions. They fall into one or more of the groups in the said brackets. The classification is usually for either regulatory purposes or for clarity of technical activities in the area [5].

\section{Approach}

Regarding the way the scientist approached in the development process, vaccines can be either killed or live. This decision is reached after answering plenty of technical questions such as how the immune system responds to the germ? Who is to be vaccinated? And which technology to use for that purpose [6].

\section{Liquid and cake}

Live vaccines: Sometimes referred to as live-attenuated or simply attenuated, these are prepared by weakening the germ that causes the infection. These vaccines contain the whole germ which is attenuated - hence the term attenuated - to the point that it cannot cause disease on a healthy target. [7]. Because the germ is viable except weakened, the body responds to it as if the bacteria or the virus is actually creating disease, hence creating high level of immunity. Because of this very background, live vaccines are very strong and known to ignite long-time immunity; literally, they are the best to prevent infectious diseases in the history of medicine. Majority of well-known veterinary vaccines including Anthrax, Rabies, Newcastle and Lumpy Skin Disease vaccines are known to mimic immunity for a year, years or even for life [7-9]. However, they are not without risk. The most serious problem associated with them is harassing the immune system. Due to underlying illness, drug treatment, pregnancy or similar reasons, the immunity of the host might have been pre suppressed. In such instances, germs in live vaccines, if administered, may multiply in high numbers and cause serious diseases [10], suggesting the risk of disease transmission than prevention [11].

Another risk is with its handling which should be viewed from two important dimensions, the first of which is the germ itself. Some organisms are highly fragile and therefore prone to easy destruction with little variations in environmental settings. Thus, they are required to be kept under high level of precautions including uninterrupted refrigeration - the cold chain principle. On the other perspective, some dangerous live organism from the vaccine, say B. Anthracis or FMD virus, if escaped to the environment, it is obvious that they can cause devastation to the animal and human health [12-14].

Inactivated: The whole germ is killed using, usually, heat or chemical, hence the term killed vaccine. Because the germ already stopped living, killed vaccines can't mimic as strong immune responses as the live ones can. For this reason, several doses and boosters may be needed before reaching to the required serological level of antibody [15]. This is particularly important when dealing with vaccine quality testing using live animal challenge test for assuring of the vaccine efficacy $[16,17]$. Compared to the previous group, they have pros, however. First, they are safe. This means that - in principle even immunosuppressed targets can be administered without fear of other complications. In practice, however, a chemical that are used to inactivate the germ are usually causing shock and significant level of morbidity and mortality in animals.

\section{Subunit}

As the name suggest, vaccines that fall in this class are prepared using some portion of the germ that can cause the immune response. These types of vaccines do not contain any whole microorganism. The parts are the most response mimicking portions usually referred to as antigens. Because of these characteristics, these vaccines sometimes are also referred to as acellular - meaning not containing any whole cells, or Purified antigens [18-20].

In principle, Subunit vaccines are killed vaccines. The major demarcation among them is whether containing the whole microorganism or not. Hence, the practical differences lie on the safety and immune response initiating ability - antigenicity, technically. Only, those parts of the microorganism - the bacteria or the virus, are incorporated as part of the vaccine, suggesting that all unnecessary artifacts are disregarded from introduction to the body of the animal. Therefore, the animal will be even safe than being administered killed vaccine $[6,7,21]$.

But there existed a strong debate whether subunit vaccines have better immunogenicity that whole killed vaccines. The favor comes from those who believe the extraction of the key part helps to concentrate into potent content. However, significant portion of the literature are against this belief. They contend that multiple dose and adjuvant supplementation are required to spark immunity [18,20-22].

Still another objection is that they are very costly due to the fact that the high level of precision requires huge amount of budget and expertise [23,24]. On top of that, the adjuvant which is used to increase the potency again in cost [25]- which elevates the vaccine budget more.

Based on the substance base of the specific antigenic portion, subunit vaccines are further classified as toxoid vaccines, polysaccharide vaccines, conjugate vaccines or recombinant vaccines - each with its definitive characteristics arisen from the chemical foundation $[22,26]$.

\section{Based on organismal origin}

Traditionally, veterinary vaccines are of either viral or bacterial - mainly. Yet, the current advancements established plant-made animal vaccines [27], insect vaccines [28], and cancer vaccines [29], which is radically changing the landscape. 
This is a revolutionary development which can challenge even the very principle of vaccine that contends "vaccines are the other forms of antigens which cause the infection". Each has its distinguishing feature which, in turn, determine the development process, quality testing technique, and handling and administration.

Bacterial vaccines: Bacteria are one of the richest sources of vaccine, as they are the major cause of infectious diseases. Bacterial vaccines are those derived from Bacteria which vaccines can include subunit vaccines as long as they are derived from bacteria [30].

The common bacterial vaccines in veterinary settings include Anthrax vaccine, Black Leg vaccine and other serious disease vaccines. Sometimes, Contagious Bovine Pleuro Pneumonia vaccines (from Mycoplasma mycoides) are classified, whereas other studies have objection with the classification.

Bacterial vaccines are very diverse - whole or subunit, live attenuated or killed, liquid or lyophilized, polyvalent, or monovalent, etc. Despite its popularity, some researchers argue that bacterial vaccines, in some extreme cases, can be causes of drug resistance. This is in instances when the host immune system is weakened and the live bacteria in the vaccine multiplied in larger numbers. In such cases, the bacterial strain for which vaccine was developed, gains resistance to the drug treatments that the host was taking. This thinking, however, is dominated by the highly recognizable importance of vaccines for drug resistance [31].

Viral vaccines: Viral veterinary vaccines are those vaccines from viruses who have veterinary importance. Viral veterinary vaccine production was driven by discovery of antigen gene discovery [32]; therefore, it is relatively a recent development in the area. In practice, veterinary viral vaccines are much more available for use than the human side of medicine - the reason being unclear [33].

As are Bacterial vaccines, viral vaccines can be live attenuated or inactivated [34]. Based on the viral nature, viral vaccines can further be classified as viral RNA vaccine and viral DNA vaccine [35]. This classification is based on the nature of the virus; RNA virus are those viruses with single stranded nucleic acid unlike DNA viruses which have double stranded nucleic acid. The common viral vaccine in veterinary setting include Rabies vaccine, Lumpy Skin Disease vaccine, Pox vaccine and others.

\section{Based on dosage formulation and combination}

Liquid versus cake: Cake vaccines are freeze died vaccines or lyophilized vaccines. They are usually in solid state and they are required to be reconstituted before administration to the target animal. The lyophilization process requires strong precautions and high cost technology - basically, the lyophilizer. The common vaccines in this category is Anthrax, among others.
On the other hand, liquid vaccines are those vaccines with liquid state presentation. Usually, vaccines in this category are killed (although live liquid vaccines are available). This is mostly pertinent to the liquid chemicals used to inactivate the pathogenic microorganism - usually formalin.

\section{Monovalent versus polyvalent}

Sometimes target pathogens can be found to be subdivided into serotypes which individually cause specific diseases. Polyvalent vaccines are mixed serotype specific immunogens, to as to achieve broader protection [36,]. Sometimes the scope of polyvalent vaccine goes far beyond serotypes of a certain bacteria. Microorganisms with similar characteristics sometimes cause diseases which are preventable with a polyvalent vaccine. However, this must not be confused with vaccine combination, which is a different concept.

On the other hand, monovalent vaccines are vaccine that target to a single individual disease that is caused by a certain pathogenic microorganism [37].

\section{Vaccine development process}

Vaccine development requires completion of lots of processes which make the process complex, twisty, and lengthy. Sets of activities in assessment of the priority areas, basic laboratory research, clinical research, and pilot production, full scale production, and post clinical assessment all makeup the helical journey of vaccine development. The process begins with years of research in laboratories aiming to ensure the next work is supported by theoretical reality [38]. In fact, vaccine development is a multidisciplinary process with plenty of nodes across the way. In practice, the stages of vaccine development are not neatly divided and not orderly staked, either. More to that, type of vaccine, status of the target disease, national policies and regulations, technological capabilities add to the complexity.

Due not only to the high recurrence of infectious pathogens, but also to the economic implications of vaccine, countries are entering to its attention, and setting standards for their projects. Recently, the UK Vaccine Research and Development Network developed a veterinary vaccine development process map which includes five broad segments - each with complicated activities requiring multidisciplinary involvement for veterinary vaccines [39]. The map (Figure 1) summarizes the process from the clinical and regulatory perspective.

Target Product Profile (TPP) is all about the project plan and foundation establishment. It is a strategy setting stage where goals are set, team characteristics are specified, and workflow developed along with all the financial and other resources. According to the network, a well-developed TPP should beware of, at least, the following checklist:

Project title

Target Antigens 


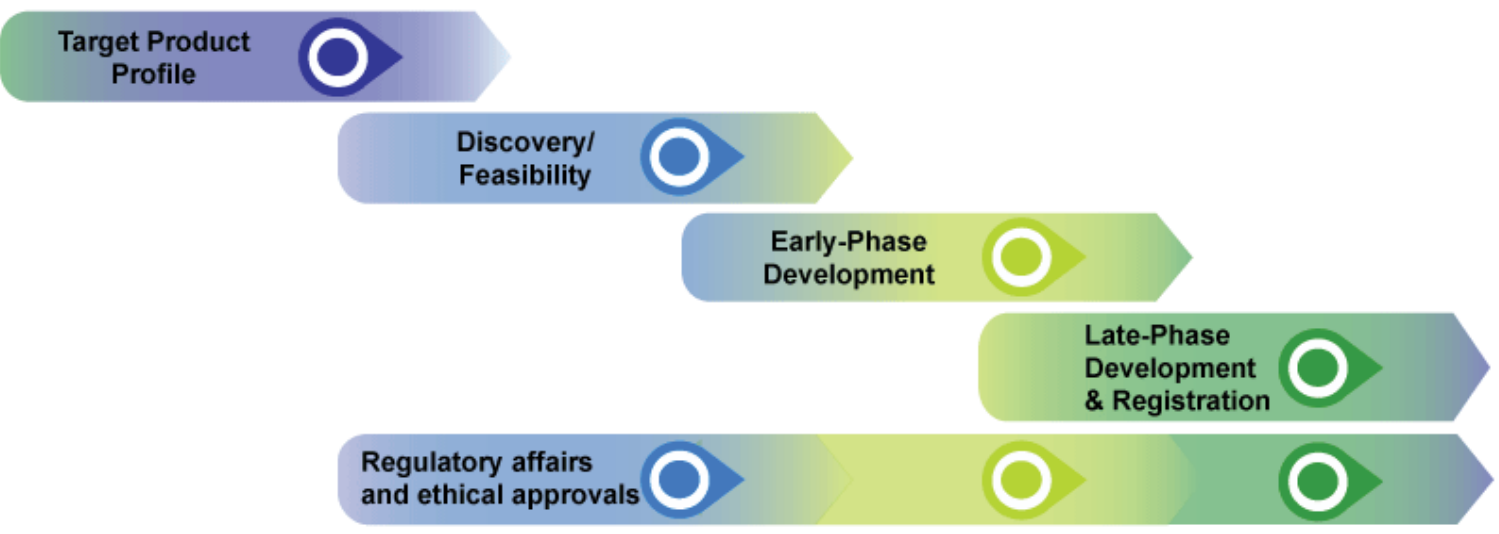

Figure 1: Veterinary Vaccine Development Process Map. Source: Vaccine Research \& Development Network

Objective/Indication for use (usage scenario- emergency/ reactive or prophylactic/ preparedness, risk/benefit profile, etc.)

Disease Prevention Claims (prevent/reduce, clinical signs/ infection)

Target Species (the target species, and sub-category), Minimal Age for Vaccination

Dosing Schedule (single/multiple doses, time between doses, booster requirements etc.)

Route of Administration (injectable - intramuscular/ subcutaneous, non-injectable, oral delivery etc.)

Onset of Immunity, Duration of Immunity, Safety Claims (e.g. safe in pregnant animals, safety in lactation or lay)

Adverse Reactions (swelling at vaccination site, side effects, frequency, etc.)

Product stability and storage (shelf life, temperature requirements, preservatives, necessity/desirability/ability to stockpile etc.)

Withdrawal Period, Immunological Properties (mode of action), Co-administration with other vaccines (interference with other vaccines etc.)

Interaction with other medicinal products, Presentation (liquid/lyophilized, mono-dose/multi-dose, dosagevolume etc.)

Formulation (vial size, concentration, fill volume etc.)

Production (number of doses required over what period of time, ability to scale up/out, high/low volume supply, affordability etc.)

Registration Strategy (type of license), Marketing attributes (Understanding the level of commercial interest, competitive advantage relative to competitor products, cost to produce versus anticipated sale price etc.)
Post marketing surveillance (monitoring of safety, protection of the target population, vaccine breakdowns, etc.)

The next stage, which the Network refer to as Discovery/ Feasibility, is a more complex dive where multiangle evaluation of the process is performed regarding its financial, technical, regulatory, and biomedical soundness of the process (Figure 2). It is a kind of feasibility study which tries to ensure that the project is worth of further effort and investment. It is base for the next phase which is a gradual dive into the actual lab work.

The third step in the map is early phase development which is the actual development work. Budding out from the discovery phase, this phase goes from Lead identification all the way through to the field trial application. lead identification, lead development, re-cloning strain development and antigen presentation, vaccine characterization, formulation and adjuvantization, process development, in vivo and in vitro efficiency tests, quality control and stability testing are all in this phase.

The fourth phase is the late phase development and registration. It is a broad categorization of activities incorporating several steps in trial and legalization process. Here, safety management related activities and immunogenicity assessment are performed along with scaling and manufacturing procedures.

The fifth phase is of regulatory aspect. Based on the UK's perspective, the Network describes the ethical and corporate procedures the projects should meant to have. This is common to other countries with slight differences. The FDA of the United States, APVDFQAC (Animal Products, Veterinary Drugs and Feed Quality Assessment Center) of Ethiopia, and other respective bodies of other countries do the same regulatory framework in their own settings. Almost all countries across the globe has lengthy and strict regulation towards the process, suggesting that vaccines are not approved as demanded and this leads to imbalance. 


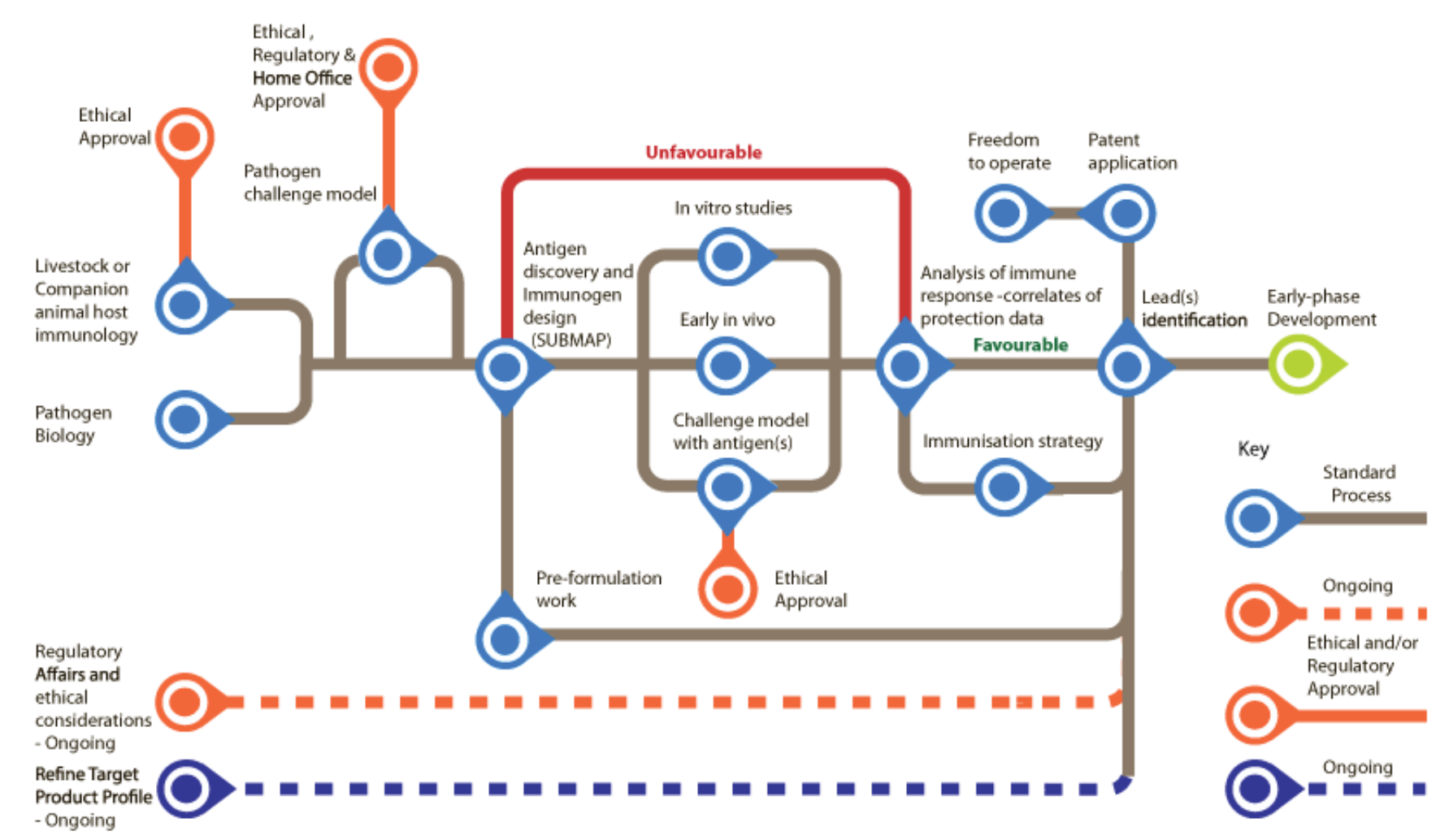

Figure 1: Veterinary Vaccine Development Process Map. Source: Vaccine Research \& Development Network

The Canada government set out a three-stage process framework for development of both human and veterinary vaccines. In the first stage where they focus on prioritization and foundation setting, priority setting regarding the risks associated with human and animal health, antimicrobial resistance and the disease burden are required to be considered. On the second stage aims at current pertinent threat in the country and technological possibilities to go through. And in the final stage, issues related to clinical and safety aspects considered [40].

More to that international organizations such as the World Health Organization (WHO), the World Organization for Animal Health (OIE), the Center for Disease Control (CDC), and Food and Agriculture Organization of the United Nations (FAO) set standards and recommendations on vaccine development and regulation [41-43].

\section{References}

1. CDC. Vaccines: The Basics. 2020. https://www.cdc.gov/vaccines/vpd/ vpd-vac-basics.html

2. Merriam Webster. Biological. 2020. https://www.merriam-webster. com/dictionary/biological

3. WHO. Biologicals. 2020. https://www.who.int/health-topics/biologicals/ tab=tab_1

4. Winchester Hospital. Health Library. 2020. https://www. winchesterhospital.org/health-library/article?id=222982

5. The College of Physicians of Philladelphia. Different Types of Vaccines. 2020. https://www.historyofvaccines.org/content/articles/different-types-vaccines
6. HHS. Vaccine Types. 2020. https://www.vaccines.gov/basics/types

7. Oxford Vaccine Group. Types of vaccines. 2020. https://vk.ovg.ox.ac. uk/vk/types-of-vaccine

8. National Veterinary Institute. Live Newcastle vaccine. 2020. https:// www.nvi.com.et/products/vaccines-against/poultry-diseases/livenewcastle/

9. Dai X, Xiong Y, Jian NL. Vaccine Types. 2019. https://www.intechopen. com/books/vaccines-the-history-and-future/vaccine-types

10. Bass P. What You Need to Know About Live Virus Vaccines. 2020. https://www.verywellhealth.com/what-is-a-live-virus-vaccine-200925

11. Asociation the conversation ESPAÑA. Live attenuated vaccines: should we rethink vaccination strategies? 2019. https://theconversation. com/live-attenuated-vaccines-should-we-rethink-vaccinationstrategies-123492

12. WebMD LLC. Development of Vaccines Toward the Global Control and Eradication of Foot-and-mouth Disease. 2020. https://www.medscape. com/viewarticle/740466_2

13. CDC. Anthrax Vaccination: What Everyone Should Know. 2020. https:// www.cdc.gov/vaccines/vpd/anthrax/public/index.html

14. Drug UF. Anthrax. 2020. https://www.fda.gov/vaccines-blood-biologics/ vaccines/anthrax

15. Carringtone College. Different Types of Vaccines. 2015. https:// carrington.edu/blog/different-types-of-vaccines-2/

16. WHO. Vaccine quality. Retrieved from Immunization standards. 2020. https://www.who.int/immunization_standards/vaccine_quality/en/

17. OIE. OIE terresterial manual. OIE. 2018.

18. Creative Biolabs. Subunit Vaccines. 2020. https://www.creativebiolabs.com/vaccine/subunit-vaccine-design.htm

19. Lidder P, Sonnino A. Biotechnologies for the Management of Genetic Resources for Food and Agriculture. Advances in Fenetics. 2016. 
20. National Institute of Allergy and Infectious Diseases. Vaccine Types. 2019. https://www.niaid.nih.gov/research/vaccine-types

21. Ministry Health System. Types of vaccines. 2020. https://www. health.mil/Military-Health-Topics/Health-Readiness/ImmunizationHealthcare/Clinical-Consultation-Services/Types-of-Vaccines

22. The immunization advisory center. Types of vaccines. 2020. https:// www.immune.org.nz/vaccines/vaccine-development/types-vaccines

23. WHO. WHO finds India's vaccine regulatory authority compliant with international standards. 2020. https://www.who.int/medicines/ regulation/india-authority_reg_compliant-int-standards/en/

24. Endert P. Antigen Processing (2nd ed.). New York: Springer Science+Business Media, LLC. 2019.

25. Fox CB.. Vaccine adjuvants. New York: Humana Press. 2017.

26. Ferran MC, Skuse GR. Recombinant Virus Vaccines. New York: Springer Science+Business Media LLC. 2017.

27. MacDonald J. Prospects of Plant-Based Vaccines in Veterinary. Cham: Springer International Publishing. 2018.

28. Thomas S. Vaccine Design (2nd ed., 2). New York: Springer Science+Business Media. 2016.

29. Lukashevich IS, Shirwan H. Novel Technologies for Vaccine Development. London: Springer-Verlag. 2014.

30. Giesker K, Hensel M. Types of Bacterial Vaccines. Sience Direct. 2014. https://www.sciencedirect.com/topics/medicine-and-dentistry/ bacterial-vaccine

31. David E, Bloom SB. Antimicrobial resistance and the role of vaccines. PNAS. 2018.

32. Jorge S, Dellagostin OA. The development of veterinary vaccines: a review of traditional methods and modern biotechnology approaches. Biotechnol Res Innov. 2017. 6-13.
33. Baron MD, Iqbal M, Nair V. Recent advances in viral vectors in veterinary vaccinology. Current opinion in virology. 2018. 1-7.

34. WHO. (n.d.). Veterinary Vaccines. Retrieved from https://www.who.int/ rabies/animal/veterinary_vaccines/en/

35. Emory University. What is Viral Vector Vaccines? 2019. http://globalhealthprimer.emory.edu/targets-technologies/viral-vectorvaccines.html

36. Schlingmann B, Castiglia KR, Stobart CC, Moore ML. Polyvalent vaccines: High-maintenance heroes. (K. R. Spindler, Ed.) 2018.

37. WHO. Types of vaccine. 2020. https://vaccine-safety-training.org/ types-of-vaccine.html

38. CHOP. Making Vaccines: Process of Vaccine Development. 2020. https://www.chop.edu/centers-programs/vaccine-education-center/ making-vaccines/process-vaccine-development

39. UK Vaccine Network. (n.d.). Welcome to the Vaccine Development Process Map. 2020. http://vaccinedevelopment.org.uk/

40. Government of Canada. (n.d.). Vaccine research and development priorities. https://www.canada.ca/en/public-health/services/vaccineresearch-development-priorities.html\#a1

41. WHO. Subunit vaccine. 2020. https://vaccine-safety-training.org/ subunit-vaccines.html

42. FDA. The Division of Biological Standards and Quality Control (DBSQC) within OCBQ has many responsibilities, including the following. 2018. https://www.fda.gov/vaccines-blood-biologics/guidance-complianceregulatory-information-biologics/division-biological-standards-andquality-control-dbsqc

43. OIE. Avian Influenza Portal. 2020. https://www.oie.int/en/animalhealth-in-the-world/avian-influenza-portal/prevention-and-control/ 